\title{
Bestrittene Honorarforderungen im Inkassoverfahren - der Gang vor die kantonalen Gerichte
}

\author{
M. Fankhauser
}

\section{Einleitung}

Wird eine Honorarrechnung trotz Mahnung(en) nicht bezahlt, kann die Forderung in Betreibung gesetzt werden. Gemäss dem schweizerischen Schuldbetreibungs- und Konkursgesetz (SchKG) bedarf es dazu eines Anstosses durch den Rechtsuchenden ( = Gläubiger), indem dieser das Betreibungsbegehren einleitet. Nach Empfang des Betreibungsbegehrens erlässt das Betreibungsamt den Zahlungsbefehl. Ob der Gläubiger berechtigt ist, den im Betreibungsbegehren geltend gemachten Anspruch zu erheben und ob der Anspruch vollstreckbar oder überhaupt materiellrechtlich begründet ist, prüft das Betreibungsamt nicht. Daraus folgt, dass jedermann auch ohne rechtliche Grundlage - jemanden betreiben kann. Diese Eigenart unseres Gesetzes macht es notwenig, dem Schuldner die Möglichkeit zu geben, sich zur Wehr zu setzen.

Der Betriebene kann innert 10 Tagen seit Zustellung des Zahlungsbefehls «Rechtsvorschlag» in der gegen ihn gerichteten Betreibung erheben. Mit dem Rechtsvorschlag bestreitet der Schuldner, dass die betriebene Forderung oder ein Teil derselben zu Recht besteht. Er erhebt gewissermassen Einspruch gegen die Betreibung. Allzu häufig wird diese Einsprache jedoch (nur) erhoben, um das Betreibungsverfahren zu verzögern oder den Gläubiger zu ärgern. Immer wieder gehen Patienten ausserdem davon aus, dass der Arzt des geringen Streitwertes wegen kein Gerichtsverfahren anstreben wird.

Da in der Regel der Schuldner nach Erhebung des Rechtsvorschlages nicht freiwillig zahlt und das Betreibungsverfahren aber solange stillsteht, wie der Rechtsvorschlag besteht, muss dieser beseitigt werden. Die Beseitigung eines Rechtsvorschlages bedeutet, dass die Forderung auf dem Zivilprozessweg bewiesen werden muss. Der Schuldner muss gerichtlich dazu verurteilt werden, den Ausstand zu begleichen. Je besser die Beweismittel (das Gesetz kennt hier klar definierte Voraussetzungen), um so schneller kann ein Rechtsvorschlag beseitigt werden. Forde- rungprozesse aus ärztlichen Honorarrechnungen führen nun regelmässig auf dem «ordentlichen Prozessweg» vor die kantonalen Gerichte, weil die «Honorarrechnung» an sich kein genügendes Beweismittel für das Bestehen der Forderung darstellt. Dieses Verfahren steht, im Gegensatz zum eigentlichen (formellen) Rechtsöffnungsverfahren (siehe Ziffer 3), ausserhalb der Schuldbetreibung. Seine erfolgreiche Durchführung ist aber ebenfalls Voraussetzung für den Fortgang der Betreibung. Das im Zivilprozess ergangene Urteil ermächtigt den Gläubiger, ohne weiteres die Fortsetzung der Betreibung zu verlangen.

\section{Ordentliches Verfahren}

Die Zivilprozessordnungen variieren von Kanton zu Kanton und auch die Zulassung von Vertretungen und Bevollmächtigten ist daher nicht überall gleich. In der Regel beginnt der ordentliche Prozessweg mit einer Sühneverhandlung vor dem für den Betreibungsort zuständigen Friedensrichter, welche der Gläubiger beantragen muss. Schuldner und Gläubiger haben persönlich an der Sühneverhandlung zu erscheinen; die Vertretung - z. B. durch ein Inkassobüro - ist praktisch nicht möglich. Sehr oft wird aber auf diesen Schritt verzichtet, sei es, weil das KostenNutzen-Verhältnis unstimmig ist oder weil gerade der Arzt/die Ärztin oft vor einer Hemmschwelle stehen und/oder weil Zeit/Lust und das Wissen fehlen, das richtige Verfahren einzuleiten. FMH Inkasso Services kann zwar keinen ihrer Auftraggeber vor Gericht vertreten. Sie fertigt aber gegen einen Unkostenbeitrag von Fr. 100.- die für den jeweiligen Einzelfall richtige Rechtsschrift vollständig aus, der Arzt/die Ärztin muss sie nur noch datieren, unterzeichnen und einsenden. Für die mündliche Verhandlung wird ein sog. «Argumentarium» ausgearbeitet, so dass der Arzt/die Ärztin das Rechtsbegehren ohne weiteres vertreten kann. 


\section{Rechtsöffnungsverfahren}

Kann der Gläubiger seine Forderung durch eindeutige Dokumente beweisen, so kann er den Rechtsvorschlag in einem abgekürzten Verfahren beseitigen lassen. In der Fachsprache nennt man dies ein «summarisches Verfahren», der richterliche Entscheid heisst Rechtsöffnung. Je nach Qualität dieser Dokumente kann die «provisorische» oder «definitive» Rechtsöffnung beantragt werden. Rechtsöffnungsverfahren werden in der Regel ohne Anwesenheit der Parteien durchgeführt; der Richter entscheidet aufgrund der Akten.

\section{Definitive Rechtsöffnung}

Unter der definitiven Rechtsöffnung ist der richterliche Entscheid zu verstehen, der aufgrund eines gegebenen vollstreckbaren Urteils die Einstellungswirkung des Rechtsvorschlages gegen den Zahlungsbefehl endgültig beseitigt. Solche «Rechtsöffnungstitel» sind gemäss Art. 80 SchKG ausschliesslich, vollstreckbare, gerichtliche Urteile, diesem gleichgestellte Urkunden (z. B. gerichtlicher Vergleich, gerichtliche Schuldanerkennung). Liegt ein solcher Vollstreckungstitel vor und erhebt der Schuldner im Rechtsöffnungsverfahren keine Einwendungen oder werden seine Einwendungen als unbegründet erklärt, so heisst der Richter das Gesuch des Gläubigers gut und erteilt ihm die Rechtsöffnung. Mit diesem rechtskräftigen Entscheid ist der Rechtsvorschlag ein für allemal beseitigt; dem Fortgang des Betreibungsverfahrens steht nichts mehr im Wege.

\section{Provisorische Rechtsöffnung}

Unter provisorischer Rechtsöffnung versteht man den richterlichen Entscheid, der auf Grund einer schriftlichen Schuldanerkennung die betreibungshemmende Wirkung des Rechtsvorschlages bloss bedingt aufhebt, indem er noch die Nachprüfung des materiellen Bestandes der Forderung durch den ordentlichen Richter vorbehält (= Aberkennungsklage). Die Schuldanerkennung muss schriftlich festhalten, dass der Schuldner dem Gläubiger einen bestimmten Forderungsbetrag schuldet; die Anerkennung darf keiner Bedingung unterliegen und muss vom Schuldner unterzeichnet sein.

Die Wirkungen der provisorischen Rechtsöffnung gehen somit bedeutend weniger weit als diejenigen der definitiven. Das hängt damit zusammen, dass ihre Voraussetzungen weniger streng sind als diejenigen der definitiven. Für die provisorische Rechtsöffnung genügt es, dass der Gläubiger eine Schuldanerkennungsurkunde vorweist, die keinerlei autoritative Aussagekraft, sondern bloss einen gewissen Wahrscheinlich- keitswert besitzt. Ein Rechtsöffnungstitel im Sinne von Art. 82 SchKG stellt regelmässig der «Verlustschein infolge Pfändung» dar; der Verlustschein infolge Konkurs nur dann, wenn der Schuldner die Forderung im Konkursverfahren anerkannt hat!

\section{Überlegungen für / gegen ein Gerichtsverfahren}

Ein obsiegendes Urteil garantiert noch nicht, dass die Fortsetzung der Betreibung erfolgreich sein wird; der Entscheid über die Einleitung eines Zivilprozesses oder eines Rechtsöffnungsverfahrens ist immer im Einzelfall zu fällen und die Solvenz des Schuldners genau zu prüfen. Oft scheuen sich Gläubiger, aus verständlichen Gründen, ihre Guthaben gerichtlich durchzusetzen. Ein Gerichtsverfahren kann mit erheblich höherem Kosten- und Zeitaufwand verbunden sein, als dies bis zum Zeitpunkt des zugestellten Zahlungsbefehls der Fall war. Wenn der Schuldner jedoch feststellen muss, dass der Gläubiger auf der Bezahlung des ausstehenden Betrages besteht, so wird er sich vielleicht überlegen, ob er die doch recht hohen Gerichtskosten auch noch zusätzlich zahlen will. Andererseits sollen notorische Nichtzahler und Leute, die immer wieder auf diese Art um die Bezahlung ihrer Schulden kommen, nicht in jedem Fall verschont werden. Immerhin geht es ja um die Durchsetzung einer Forderung, für die der Arzt/ die Ärztin Leistungen erbracht hat und er muss ebenso wie alle Gläubiger von Geldforderungen zunächst den Weg über das Schuldbetreibungsund Konkursgesetz, und im Falle der Bestreitung seiner Forderung, den ordentlichen Prozessweg, allenfalls auf das Rechtsöffnungsverfahren, gehen; er kann also gar nicht anders vorgehen, wenn er zu seinem Geld kommen will.

\section{Arzt-Patienten-Geheimnis}

Für die Ärzteschaft spielt im Prozessfall aber immer auch das Arzt-Patienten-Geheimnis eine aussergewöhnlich wichtige Rolle. Medizinische Daten sind besonders sensible Daten, deren Bearbeitung und Weitergabe durch die Datenschutzgebung und das Strafrecht geregelt bzw. geschützt werden. Hat der Arzt/die Ärztin den Umgang mit medizinischen Daten nicht schon bei Behandlungsbeginn mittels einer sog. Patientenerklärung geregelt, muss er sich vor jedem Gerichtsverfahren durch die zuständige kantonale Gesundheitsbehörde von der ärztlichen Schweigepflicht entbinden lassen! 


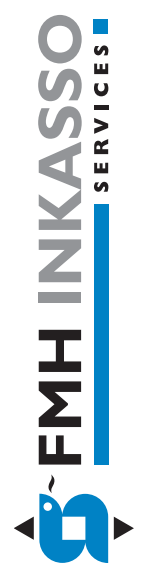

Ihr Partner, wenn Zahlungseingänge sich verzögern!

Säumige Patientinnen und Patienten? Wenn die Zahlungsmoral krankt, ist Erfahrung gefragt. Wir sind die Spezialisten, entlasten Sie von Inkassosorgen und treiben für Sie die Honorarforderungen ein. Und all dies zu fairen Konditionen, mit grosser Sensibilität und mit der gefragten Kompetenz. Damit Sie sich aufs Wesentliche konzentrieren können. Zum Wohl Ihrer Patientinnen und Patienten.

\section{Votre partenaire pour le règlement de vos factures!}

Avez-vous des patients mauvais payeurs? Si cela vous irrite, faites appel à notre expérience. Nous sommes des spécialistes, nous nous occupons de vos soucis de contentieux et encaissons pour vous les honoraires dus! Et tout ceci à des conditions équitables, avec tact et compétence. Pour que vous puissiez vous concentrer sur l'essentiel. Pour le bien des vos patients et patientes.

FMH Inkasso Services, Thorackerstrasse 3 , 3074 Muri b. Bern,

Tel. 03195080 30, Fax 0319508040

E-Mail: mail@fmhinkasso.ch 